ISSN: 2602-8506

Vol. 4, $\mathrm{N}^{\circ} 1 .$, p.06-22, enero - marzo, 2020

Recibido: 02-11-2019/Aceptado: 04-12-2019/ Publicado: 04-01-2020

\title{
Plan estratégico para la contaminación ambiental en el sector del arenal de la ciudad de Cuenca-Ecuador
}

\section{Strategic plan for environmental pollution in the sand sector of the city of Cuenca- Ecuador}

Kleber Antonio Luna Altamirano. ${ }^{1}$, Jennifer Gisela Yanza Nantip. ${ }^{2}$, Domenica Estefanía Miguitama Gonzalez. ${ }^{3} \&$ Gissella Magdalena Plasencia Sagbay. ${ }^{4}$

DOI: https://doi.org/10.33262/visionariodigital.v4i1.1064

\begin{abstract}
The Arenal sector located in the city of Cuenca-Ecuador, where a market is located to sell basic products that make up the family basket, this place is known as Free Fair. The problem of this research is the excess of solid waste derived from commercial activity within and outside this market, due to the lack of intervention of organizations in charge of the environmental wellbeing of the city. The objective is to design and present a strategic plan to the regulatory health control authorities and institutions to try to minimize solid waste contamination in the mentioned sector. The research is of a descriptive type with a quantitative approach, where the deductive method will be applied. For this purpose, a survey will be developed aimed at the suppliers of the products of basic need and citizenship through a questionnaire. The results show fewer unsorted garbage cans and less support from institutions in charge of environmental ordinances, as well as excess solid waste thrown in and out of this sector. With the contribution of this research it will be possible to recover the ornament of this place, and with it to minimize the environmental pollution, coming to the citizenship to raise awareness in the maintenance and cleanliness of this zone of sale of products of first need.
\end{abstract}

\footnotetext{
${ }^{1}$ Doctor (c) en Ciencias Sociales Mención Gerencia, Universidad del Zulia República Bolivariana de Venezuela, profesor investigador Unidad Académica de Administración Universidad Católica de Cuenca, klunaa@ucacue.edu.ec

${ }^{2}$ Estudiante de la carrera de Contabilidad y Auditoría en la Unidad Académica de Administración de la Universidad Católica de Cuenca, jennifer.yanza.65@est.ucacue.edu.ec

${ }^{3}$ Estudiante de la carrera de Contabilidad y Auditoría en la Unidad Académica de Administración de la Universidad Católica de Cuenca, domenica.miguitama.36@est.ucacue.edu.ec

${ }^{4}$ Estudiante de la carrera de Contabilidad y Auditoría en la Unidad Académica de Administración de la Universidad Católica de Cuenca, gissella.plasencia.05@est.ucacue.edu.ec
} 
Keywords: Strategic plan, environmental pollution, survey, excess solid waste

\section{Resumen}

El sector Arenal ubicado en la ciudad de Cuenca-Ecuador, en donde se encuentra localizado un mercado de expendio de productos de primera necesidad que conforman la canasta familiar, a este lugar se le conoce como Feria Libre. El problema de esta investigación es el exceso de desechos sólidos derivados de la actividad comercial dentro del interior y exterior de este mercado, por la falta de intervención de organizaciones encargadas del bienestar ambiental en la ciudad, hace que la presentación de este sector cree un impacto desfavorable para la ciudadanía. El objetivo es diseñar y presentar un plan estratégico a las autoridades e instituciones reguladoras de control sanitario para tratar de minimizar la contaminación de desechos sólidos en el sector mencionado. La investigación es de tipo descriptiva con enfoque cuantitativo, donde se aplicará el método deductivo, para ello se desarrollará una encuesta dirigida a los proveedores de los productos de primera necesidad y ciudadanía a través de un cuestionario. Los resultados arrojan menos botes de basura sin clasificación y un apoyo insuficiente de las instituciones encargadas de ordenanzas ambientales, además de exceso de desechos sólidos arrojados dentro y fuera de este sector. Con el aporte de esta investigación se logrará recuperar el ornato de este lugar, y con ello minimizar la contaminación ambiental, llegando a la ciudadanía a concientizar en el mantenimiento y aseo de esta zona de expendio de productos de primera necesidad.

Palabras clave: Plan estratégico, contaminación ambiental, encuesta, exceso de desechos sólidos.

\section{Introducción}

La feria libre es uno de los mercados comerciales de productos alimenticios más visitados de la ciudad de Cuenca, provincia del Azuay, Ecuador. En este espacio comercial se expende la mayor parte de productos de primera necesidad que conforman la canasta familiar de la ciudadanía cuencana. El problema radica por la acumulación de desechos sólidos por parte de visitantes y mercaderes en toda esta área de comercio, creando una presentación desfavorable para la ciudadanía afectando al medio ambiente, la contaminación ambiental es un fenómeno nocivo producido por los seres humanos que afecta a todos los seres vivos en el planeta. Orozco, Pérez, González, Rodríguez y Alfayate (2003) afirman "El empleo de materiales de usar y tirar, es decir, la cultura de los bienes de corta vida media, incrementara así mismo los problemas derivados de la utilización desmesurada de recursos naturales, (...) aparición de sustancias con efectos 
Vol. 4, $\mathrm{N}^{\circ} 1 .$, p.06-22, enero - marzo, 2020

ambientales nocivos a largo plazo; es el caso de los clorofluorocarburos (CF's), causantes en gran medida del deterioro de la capa de ozono" (pp.21-22). El objetivo del estudio es diseñar y presentar un plan estratégico, el cual se considera como una herramienta que permite conseguir un progreso a través de un conjunto de acciones dirigidas hacia organizaciones determinadas, este plan está encaminado hacia las autoridades e instituciones reguladoras de control sanitario para tratar de minimizar la contaminación de desechos sólidos en el sector mencionado. Alvarado (2001) asevera "Un Plan Estratégico es un conjunto de elementos y/o conceptos que orientan, unifican, integran y dan coherencia a las decisiones que dan rumbo y destino a una organización (...)” (p.284).

En lo metodológico, la investigación es de tipo descriptivo, en la cual se establece varias características, variables y conceptos de un fenómeno determinado para describirlas independientemente, Salkind (1999) afirma "La investigación descriptiva reseña las características de un fenómeno existente. Los censos nacionales son investigaciones descriptivas, lo mismo que cualquier encuesta que evalúe la situación actual de cualquier aspecto" (p.11). El enfoque del estudio es cuantitativo, se considera como un estudio basado numéricamente para la medición, recolección de datos y resaltando el comportamiento de esquemas en una población, Hernández, Fernández y Baptista (2014) sostiene "se miden las variables en un determinado contexto; se analizan las mediciones obtenidas utilizando métodos estadísticos, y se extrae una serie de conclusiones respecto de la o las hipótesis" (pp.4-5). En cuanto al método se aplicará el deductivo, ya que especifica un razonamiento que extrae a partir de hipótesis conclusiones lógicas para deducir de manera más rigurosa todas las consecuencias posibles, Abreu (2014) asevera:

El método deductivo permite determinar las características de una realidad particular que se estudia por derivación o resultado de los atributos o enunciados contenidos en proposiciones o leyes científicas de carácter general formuladas con anterioridad. Mediante la deducción se derivan las consecuencias particulares o individuales de las inferencias o conclusiones generales aceptadas (p.200).

Se aplica como técnica de investigación, la encuesta, esta es una herramienta que se utiliza en la investigación para recopilar datos y llegar a posibles conclusiones.

La encuesta es un instrumento de captura de la información estructurado, lo que puede influir en la información recogida y no puede/debe utilizarse más que en determinadas 
situaciones en las que la información se quiere capturar está estructurada en la población objeto de estudio (Alvira, 2011, p.14).

En la presente investigación se desarrolla y aplica preguntas realizadas a obtener información relevante para el diseño del plan estratégico.

El estudio está compuesto de los siguientes ítems: En primera instancia dentro del estado del arte, se da soporte teórico con investigaciones a los autores que dan relevancia a la investigación. Como parte de la metodología se especifica la construcción de un modelo de plan estratégico que será expuesto para el mejoramiento del ornato en el sector a investigar. Consecutivamente se plantea los resultados ante la mejora de la presentación de este sector comercial brindando comodidad a los consumidores y mercaderes, como conclusión se presentará a las autoridades para reducir los desechos sólidos, con ello se logrará una solución ante el problema ambiental que afecta a este sector.

\section{Estado del arte}

Al diseñar un plan estratégico para el control de limpieza del sector arenal de la ciudad de CuencaEcuador, es de suma importancia ya que este permitirá mejorar la el ornato de la ciudad, por ello algunos autores contribuyen con sus investigaciones relacionadas al estudio del medio ambiente, entre ellos: Ramírez (2005) propone explorar dimensiones geopolíticas, las cuales podrán enriquecer a la participación ciudadana, además fomentar el uso de consulta popular y la negociación entre los involucrados relacionados con la gestión de desechos sólidos para el mejoramiento del entorno y la calidad de vida. González y Vega (2002) expresan implementar la técnica de recubrimiento, esta limita el movimiento del lixiviado evitando que fluya hasta a el subsuelo, facilita la evaporación y, en consecuencia, minimiza una posible contaminación al agua, lo que desechos se acumulen en un punto determinado y de esta manera evitar que afecte el ambiente donde habita fauna endémica de la ciudad de Mérida-Yucatán, México.

Córdova y Romo (2012) exponen una gestión del manejo para analizar la acumulación excesiva de llantas creando un riesgo para el entorno en la región fronteriza de Juárez-México, siendo este un problema ambiental porque son depositadas en tiraderos clandestinos y además se tiran a la vía pública. Ramos y Jiménez (2016) presentan un diseño de central tipo estrella para la degradación de desechos tóxicos, en el cual se integra el proceso foto-Fenton solar, este permite analizar el efecto peróxido de hidrogeno y del fierro, siendo estos desechos tóxicos y corrosivos de laboratorio 
en la Universidad de Carabobo, Venezuela. Chassaigne y Pinto (2014) proponen determinar variables respecto a la estimación de residuos, así como también de los desechos sólidos municipales, recolectados a través de un análisis multivariado, igualmente se aplica a una determinada muestra del universo en general, y los aplicados es un análisis conglomerado, correlación de Pearson y regresión múltiple dirigida al municipio de Puerto Cabello, Venezuela, para de esta manera comprender las características que muestra esta situación en la práctica. Limas y Blanco (2017) presentan estrategias de separación, generación y reciclaje para determinar las prácticas de consumo-desecho de residentes en sus domicilios de la ciudad de Juárez previo al conocer el registro de las toneladas de residuos recolectados, también aplicando el reciclaje y reduciendo su volumen a nivel local.

Por su parte Badilla, Rojas y Vargas (2008) proponen una estrategia para el desarrollo de grandes rellenos sanitarios junto con organizaciones municipales, así contribuyendo a la búsqueda de una solución del exceso de basura, se establecen lineamientos básicos para determinar la disposición de sitios de desecho considerando su ubicación, topografía e hidrogeología, se proponen tres sitios aptos en mejores condiciones físicas y de factibilidad económica al oeste del Valle central de Costa Rica. Micucci, Jarne, Ferrarotti, Munitis y Peruzzetto (2005) efectúan un estudio comparativo sobre el riesgo biológico de materiales biológicos sólidos y líquidos eliminados por pequeños centros de salud de atención primaria y de domicilios, tomando como ejemplo a pequeños laboratorios clínicos. Martínez, Tosca y Juárez (2019) presentan como objetivo analizar si existen medios para generar impuestos ambientales respecto a desechos sólidos analizando el fundamento de los impuestos en la carta Magna, el aporte de impuestos verdes en el mundo a través de la historia y el impacto de estos desechos al cambio climático. Tamajón y Quevedo (2007) proponen un estudio analítico sobre el impacto ambiental de un colector de basura de prolipropileno tomándolo desde su ciclo de vida contribuyendo a complementar el estudio que realiza el GERA (Grupo de Energías Renovables Aplicadas) de esta manera constituye un criterio extra para la selección.

En relación a planes estratégicos, los siguientes autores, aportan al mejoramiento institucional, entre ellos, Pacheco, Rodríguez y Bermúdez (2006) proponen un plan de programas de acción para contribuir a la mayor competitividad de productos hortícolas promoviendo un mejor desarrollo de 
la región de riego de Alto Chicamocha, ya que este dispone de una ubicación geográfica adecuada con respecto a los principales mercados, de esta manera se aplican diferentes estrategias como la implementación de una comercializadora teniendo a sus productores como socios, además fortalecer el conocimiento de estos productores con talleres de financiamiento como un elemento fundamental y visión productiva. Rodríguez, Morales, Villarreal y Quesada (2016) presentan un modelo de plan estratégico en mercadotecnia para el diseño de una marca que proporciona los productos elaborados que son vinculados en procesos de verificación de calidad asegurados por la institución, implementando estrategias como producto plaza, precio y promoción.

Idrovo, Luna, Ordoñez y Lituma (2019) realizan un modelo de plan de marketing para encontrar las condiciones más aptas en desarrollar una gestión económica con base y fomento del turismo local, implementando estrategias efectivas, colocando así a la Provincia Azuay-Ecuador en un centro de políticas nacionales. Stutely (2000) propone cómo elaborar un plan estratégico de negocios, el cual apoya a ejecutivos confrontados con la petición de escribir métodos y este propone un camino rápido para lograrlo, además puede ser útil para quienes pueden dedicar tiempo a un enfoque más elaborado, y para aquellas personas experimentadas de la elaboración de planes de negocios. Solano, Riascos y Aguilera (2013) plantean estrategias en sistemas de información que tienen enfoque objetivo y sistemático para una mejor toma de decisiones de pequeñas y medianas empresas ya que estas son diferentes, además a un futuro diseñar y organizar un plano de probables decisiones.

Los autores descritos, contribuyen a un mejor desarrollo de plan estratégico, ya que estos proporcionan varias alternativas para un mejor ornato y con ello llevar a cabo una estrategia para tratar de disminuir la contaminación ambiental por desechos sólidos en el sector de expendio Arenal en la ciudad de Cuenca-Ecuador.

\section{Metodología}

La presente investigación es de tipo descriptivo con enfoque cuantitativo, por lo que se aplica el método deductivo para el diseño de un plan estratégico para la contaminación ambiental en el sector arenal feria libre Cuenca-Ecuador, y la información es suministrada a través de una encuesta dirigida a 180 personas en general de este sector quienes conforman la muestra total entre comerciantes locales, comerciantes ambulantes y consumidores, con el propósito de dar mayor 
veracidad a esta información. A continuación, se detallan las personas a quienes fueron dirigidas la encuesta:

Tabla 1. Segmentación de personas en el área comercial feria libre

\begin{tabular}{lc}
\hline Total de personas & Número \\
\hline Comerciantes locales & 83 \\
Comerciantes ambulantes & 53 \\
Consumidores & 44 \\
\hline Total & $\mathbf{1 8 0}$ \\
\hline
\end{tabular}

Fuente: Elaboración propia

\section{Resultados}

Como se evidencia en la tabla 1, la muestra de la población en el área comercial feria libre de la ciudad de Cuenca, son de 180 personas a quienes se les realizó una investigación mediante la aplicación de una encuesta. Las edades de estas personas varían entre 20 a 60 años, son personas que estuvieron preestablecidos desde hace mucho tiempo en este sector de expendio y están vinculados con la problemática ambiental que presenta esta área de comercio. La información más destacada de las encuestas se presenta a continuación:

Figura 1. Propuesta estratégica

¿Cree usted que es importante que se implementara más tachos de basura en esta área comercial?

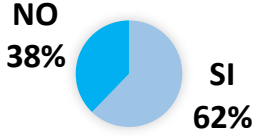

Fuente: Elaboración propia

Recopilando las respuestas basadas en el sí, con un 62\%, significa que los comerciantes locales, comerciantes ambulantes y consumidores están de acuerdo con implementar más botes de basura alrededor de esta área comercial. Entre una de las normas de reciclaje permite clasificar cada tipo de basura en varios botes de basura, Berenguer, Trista y Deas (2009) exponen que en otros países reúnen en un mismo contenedor de basura las botellas de cerveza a pesar de que posean distintas etiquetas además en Alemania y Dinamarca existen sistemas de recogidos que favorecen a la reutilización para disminuir el impacto ambiental. 
Figura 2. Propuesta estratégica

¿Le gustaria que, a los comerciantes de este sector de expendio, reciban charlas sobre la clasificación de desechos e incentivar el hábito de no botar basura? NO

$$
22 \%
$$

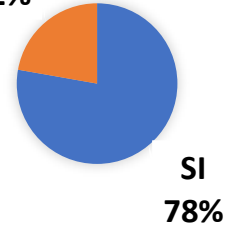

Fuente: Elaboración propia

Con relación a las charlas sobre la clasificación de desechos y el hábito de no botar la basura, un porcentaje del $78 \%$ asimila de una buena manera respondiendo con un sí, de esta manera, dando a conocer que es una muy buena idea ya que los mercaderes no conocen bastante sobre este tema. El conocimiento sobre la clasificación de los desechos y el buen hábito de no botar la basura es muy importante ya que así nos ayudará a disminuir la contaminación ambiental, asegurándonos de la reutilización de algunos materiales que se pueden reciclar. Por ello Chamán (1989) expresa que deben optimizarse los procesos, y minimizarse los volúmenes generados de residuos, debe fomentarse la cultura del reciclado e impulsar una educación adecuada del tema para conseguir una consciencia adecuada para juntos buscar y provocar un ambiente que permita proporcionar a la población una calidad de vida digna y saludable ya que el gasto y esfuerzo que esto conlleva, siempre será menor que el costo de poner en peligro el medio y la salud de los seres humanos.

Figura 3. Instituciones ambientales

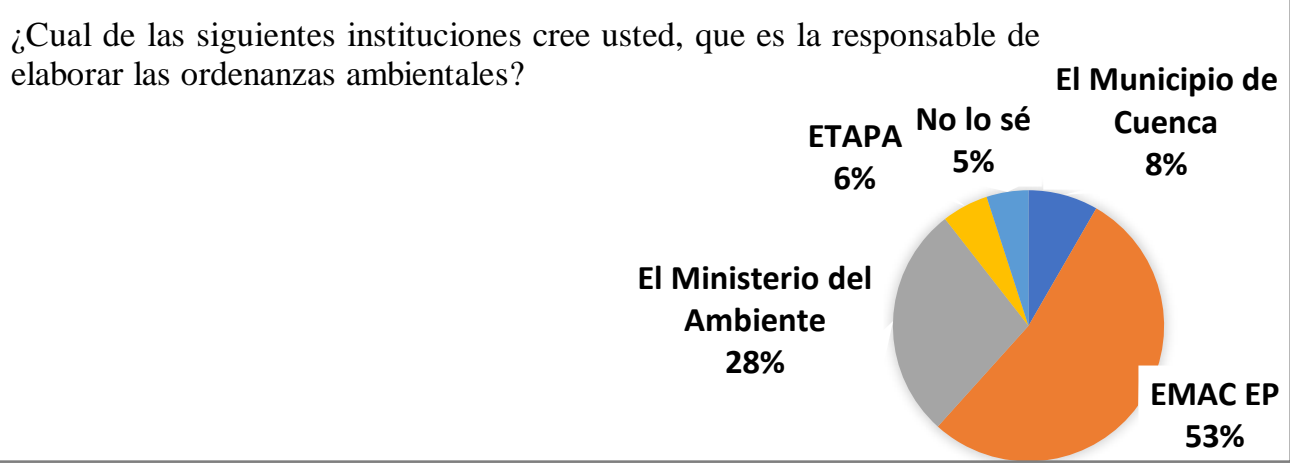

Fuente: Elaboración propia 
Respecto a las instituciones encargadas de elaborar ordenanzas ambientales un 53\% de los encuestados comentan que la organización encargada de dar ordenanzas ambientales es la EMAC EP, con esto se concluye que esta organización es la encargada del control de limpieza en la ciudad de Cuenca. La empresa pública encargada de servicio de aseo, limpieza y recolección de desechos sólidos del cantón Cuenca es la EMAC EP. Para ello La Empresa Pública Municipal de Aseo de Cuenca (EMAC EP, 2014) es la encargada de la recolección de desechos y residuos sólidos en la ciudad de Cuenca manteniendo una certificación ISO 9001 por su alta calidad de sus servicios; las industrias, mercados y centros comerciales almacenan sus desechos en contenedores de 3,28 m3 de capacidad.

Figura 4. Estado de Contaminación

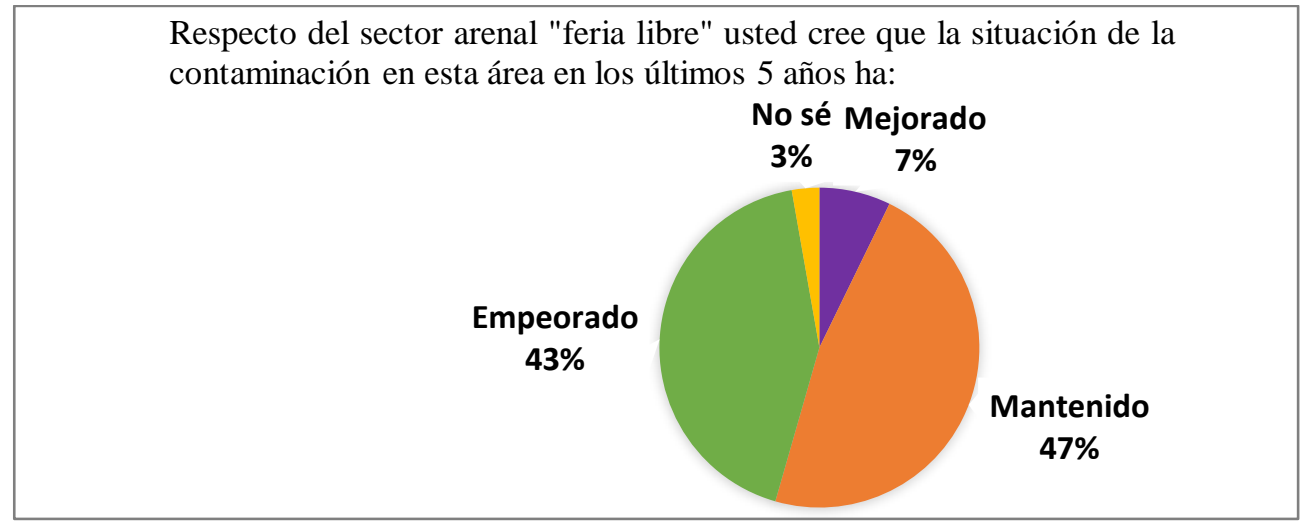

Fuente: Elaboración propia

En cuanto a la situación ambiental establece que un $43 \%$ comentan que ha empeorado y en un $47 \%$ se ha mantenido la mala presentación de este centro de expendio. Demostrando que al tener menos contenedores de basura genera que la basura se deseche en un solo bote sin clasificación ya que es un mercado comercial. Guevara (2000) afirma:

Los desechos sólidos, basuras son vertidos a contenedores sin clasificación y sólo el $7 \%$ es reciclado con indebidas formas de colecta que hacen recogedores de cartón, papel, latas y chatarras que deambulan por calles, carreteras, estacionamientos, paseos o que se concentran en vertederos, donde por lo general se confunden con roedores, buitres y otras alimañas, con grandes riesgos de contaminación a causa de la indebida mezcla de residuos químicos peligrosos con desechos hospitalarios infecto- 
ISSN: 2602-8506

contagiosos y demás tipos de basuras originadas de toda actividad comercial, pública o domiciliaria.

Figura 5. Sanciones

Cree usted que es necesario aplicar una multa a los comerciantes que alrededor de su área comercial se encuentre basulæ?

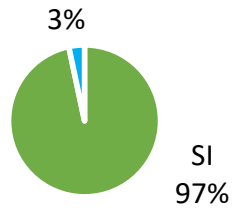

Fuente: Elaboración propia

Con respecto a las sanciones o multas dirigidas a comerciantes locales que se encuentren desechos de basura alrededor de su espacio comercial el $97 \%$ de encuestados están de acuerdo con implementar esta sanción. Vélez (2012) asevera:

las sanciones ambientales monetarias se han venido imponiendo sobre las sanciones civiles y penales de manera más frecuente, e igualmente el tamaño de las penas se ha incrementado. Los individuos, y entidades corporativas son tomados como objetivos comunes para estas sanciones, habiéndose convertido el encarcelamiento en una sanción menos común, lo mismo que las penas de prisión (p.7).

Se estructura la matriz FODA, en la cual se precisan fortalezas, oportunidades, debilidades y amenazas del sector de expendio. Es de destacar que la información para la estructura de estas matrices, se obtuvo de la misma encuesta realizada a las 180 personas que visitan y comercializan sus productos. A continuación, en la tabla 2 se indican factores internos (fortalezas y debilidades) encontradas:

Tabla 2. Matriz FODA Factores Internos

\begin{tabular}{ll}
\hline \multicolumn{1}{c}{ Fortalezas } & \multicolumn{1}{c}{ Debilidades } \\
\hline - Buena imagen en el sector. & - Plan de formación sin cumplimiento. \\
- Uso de fundas de tela. & - Presentación desfavorable. \\
- Lugar amplio y cómodo & - Personal con poca capacitación en limpieza. \\
- Estacionamiento para vehículos & - Dificultades para implementar más contenedores. \\
\hline
\end{tabular}

Fuente: Elaboración propia 
Referente a la tabla 2, sobre la matriz FODA de Factores Internos se observa que estas variables tienen como función principal demostrar cuales son los puntos fuertes del sector estudiado, en cambio las debilidades encontradas, ayudan a desarrollar nuevas ideas para robustecer la calidad del sector, en general se puede mencionar que con este análisis se puede propender a estructurar un plan eficiente con el propósito de mejorar el ornato de la ciudad.

Con la misma información, se elabora la tabla 3 que contiene los factores externos (oportunidades y amenazas).

Tabla 3. Matriz FODA Factores Externos

\begin{tabular}{ll}
\hline \multicolumn{1}{c}{ Oportunidades } & \multicolumn{1}{c}{ Amenazas } \\
\hline - Consumidores satisfechos. & $\bullet$ El sector demanda asesoramiento en aseo y recolección. \\
- Apoyo de organizaciones como el EMAC EP. & $\bullet$ Exceso de almacenamiento de desechos sólidos. \\
- Apoyo de la ciudadanía para no desechar basura. & $\bullet$ Poco personal de limpieza. \\
& $\bullet$ Enfermedades por contaminación de desechos ambientales. \\
& $\bullet$ Contaminación en productos de expendio. \\
\hline
\end{tabular}

\section{Fuente: Elaboración propia}

Una vez realizada la matriz FODA, en las variables tanto internas como externas, las cuales influyen directamente al ornato de este centro de expendio, se debe evaluar paso a paso la situación en la que se encuentra este centro internamente, se determinan las fortalezas y debilidades más relevantes, se visualiza la situación actual de esta área estudiada, cada una de estas variables permitirá tomar decisiones estratégicas que permita ver la realidad del ambiente interno para un correcto desempeño del plan estratégico del área comercial.

Esta matriz, permite visualizar la situación actual de este espacio comercial para obtener un correcto diagnóstico y tomar una decisión adecuada que permita formular un contexto que explique los lineamientos a seguir. Se presenta el Plan Estratégico, en base al análisis de la información más relevante obtenida a través de encuestas, el análisis FODA, importante para el diseño de este plan, se pretende generar cambios a nivel ambiental del sector comercial Feria Libre de la ciudad de Cuenca-Ecuador. Lo explicado a continuación (tabla 4): 
ISSN: 2602-8506

Vol. 4, N ${ }^{\circ} 1 .$, p.06-22, enero - marzo, 2020

Tabla 4. Plan estratégico ambiental

\begin{tabular}{|c|c|c|c|c|c|c|c|}
\hline Misión & Visión & Estrategias & $\begin{array}{l}\text { Optimización de } \\
\text { recursos }\end{array}$ & Protección y respeto & $\begin{array}{l}\text { Desarrollo de } \\
\text { investigación }\end{array}$ & $\begin{array}{c}\text { Acciones encaminadas } \\
\text { a minimizar el impacto } \\
\text { ambiental }\end{array}$ & $\begin{array}{c}\text { Resultados más } \\
\text { relevantes }\end{array}$ \\
\hline $\begin{array}{l}\text { La misión del centro } \\
\text { comercial Feria Libre es } \\
\text { constribuir a la } \\
\text { conservación del } \\
\text { medio ambiente con } \\
\text { solidaridad de sus } \\
\text { consumidores y } \\
\text { comerciantes } \\
\text { ejecutando estrategias } \\
\text { ecologicas. }\end{array}$ & $\begin{array}{l}\text { El centro comercial en un } \\
\text { futuro, ser un sector de } \\
\text { expendio amigable con el } \\
\text { ambiente, comprometida } \\
\text { con el mejoramiento de su } \\
\text { presentación hacia la } \\
\text { ciudadania cuencana. }\end{array}$ & $\begin{array}{l}\text { - Implementar más } \\
\text { tachos de basura. } \\
\\
\text { - Implementar multas } \\
\text { a consumidores y } \\
\text { comerciantes. } \\
\text { - Realizar charlas } \\
\text { sobre incentivación } \\
\text { de clasificación y no } \\
\text { desecho de basura. } \\
\text { - Incentivar al uso de } \\
\text { fundas de tela. } \\
\text { - Solicitar un convenio } \\
\text { entre instituciones } \\
\text { como la EMACy } \\
\text { Municipio de Cuenca } \\
\text { eara un mejor trabajo } \\
\text { ciudadanía. } \\
\text { peneficio a la } \\
\text { en varios puntos de } \\
\text { Somercialización. }\end{array}$ & $\begin{array}{l}\text { Clasificación de } \\
\text { desechos. } \\
\text { Colaboración con el } \\
\text { EMAC EP. } \\
\text {-Implementar } \\
\text { contenedores de } \\
\text { basura de diferentes } \\
\text { colores para clasificar } \\
\text { los tipos de desecho. }\end{array}$ & $\begin{array}{l}\text { Disminuir el uso de } \\
\text { las fundas plásticas. } \\
\text {. Uso de fundas de } \\
\text { tela. } \\
\text { - Efectuar } \\
\text { inspecciones para } \\
\text { verificación de la } \\
\text { gestión ambiental. } \\
\text {. Colocar anuncios de } \\
\text { reciclaje para } \\
\text { consumidores y } \\
\text { comerciantes. }\end{array}$ & $\begin{array}{l}\text { - Efectuar } \\
\text { inspecciones para } \\
\text { verificación de la } \\
\text { gestión ambiental. } \\
\text { - Realizar la revisión y } \\
\text { el seguimiento de } \\
\text { normas ambientales } \\
\text { semanalmente. } \\
\text { - Realizar un control } \\
\text { de horarios de } \\
\text { recolección de basura } \\
\text { todos los días. }\end{array}$ & $\begin{array}{l}\text { - Emitir directrices } \\
\text { jurídicas y } \\
\text { administrativa para el } \\
\text { cumplimiento de la } \\
\text { normatividad } \\
\text { ambiental. } \\
\text { - Desarrollar el Plan de } \\
\text { mantenimiento de la } \\
\text { infraestructura } \\
\text { ambiental. } \\
\text { - Apoyo de } \\
\text { fundaciones dirigidas a } \\
\text { controlar la } \\
\text { contaminación } \\
\text { ambiental en la ciudad } \\
\text { de Cuenca. }\end{array}$ & $\begin{array}{l}\text { Espacios más } \\
\text { limpios. } \\
\text { · Reducción de } \\
\text { contaminación. } \\
\text { - Incremento de } \\
\text { botes de basura } \\
\text { para reciclaje. } \\
\text { ·Multas para } \\
\text { consumidores y } \\
\text { comerciantes. } \\
\text { limpieza alrededor } \\
\text { del sector } \\
\text { comercial. } \\
\text { - Horarios de } \\
\text { recolección } \\
\text { apropiados. } \\
\text { - }\end{array}$ \\
\hline
\end{tabular}

Fuente: Elaboración propia.

En la tabla 4, se observa los cambios pertinentes que debe realizar el centro de expendio. Este plan estratégico incorpora como elementos diferenciadores de Protección y respeto y un correcto Desarrollo de investigación con la finalidad de minimizar la contaminación ambiental, una mejor presentación, implantar más contenedores en puntos estratégicos, con ello consumidores y comerciantes se sentirán agradecidos y satisfechos, se diseña este plan con base a la información obtenida de las encuestas, como se mencionó anteriormente se confeccionó esta herramienta con alrededor de 13 preguntas, las más importantes se demuestra en este estudio. Con la implantación de este plan el sector comercial mejoraría su presentación y su estado ambiental. 
Vol. 4, $\mathrm{N}^{\circ} 1$., p.06-22, enero - marzo, 2020

\section{Conclusiones}

Al diseñar un plan estratégico, para el sector comercial de expedíos Feria Libre de la ciudad de

Cuenca-Ecuador, el propósito es tratar de minimizar la contaminación por desechos sólidos y mejorar su presentación que requiere este centro de expendio. El estudio presentado en esta investigación da cumplimiento al objetivo planteado, y se orienta a mejorar de una manera más apropiada su impacto ambiental, mediante el diseño de estrategias para cada componente que conforman el plan dirigido a autoridades e instituciones encargadas del control del medio ambiente, con ello este sector comercial podrá mejorar su situación ambiental.

El tratar de reducir los desechos sólidos, representa un impacto positivo en la presentación del ornato de la ciudad de Cuenca-Ecuador, a más de ello se logrará contar con un lugar de expendio y compra de productos de primera necesidad de forma más sanitaria. El plan estratégico que se propone, se enmarca en las necesidades de comerciantes y consumidores, mediante estrategias que permitan mejorar su imagen, convirtiéndose en un centro de expendio de alimentos con mayor control sanitario, esto permitirá reducir tanto la contaminación ambiental, y que los productos sean expendidos de manera segura, con ello la sociedad cuencana propenderá a promover una nueva cultura de respeto a la preservación, cuidado y disfrute de un medio ambiente sano para el desarrollo y progreso de la ciudad.

\section{Referencias Bibliográficas}

Abreu, J. (2014). El Método de la Investigación. Daena: International Journal of Good Conscience, 9(3), 195-204.http://www.spentamexico.org/v9-n3/A17.9(3)195-204.pdf

Alvarado, T. (2001). Metodología para elaborar un plan estratégico y rediseño organizacional de una unidad de producción agropecuaria. Revista Mexicana de Agronegocios, 5(9), 284292. http://www.redalyc.org/articulo.oa?id=14100903

Alvira, F. (2011). La encuesta: una perspectiva general metodológica. España: Centro de Investigaciones Sociológicas.

https://books.google.com.ec/books?id=GbZ5JOIoDEC\&printsec=frontcover\&dq=encuest $\underline{\mathrm{a} \& h l=e s \& s a=X \& v e d=0 a h U K E w i-u-}$

\section{CXmejlAhUFw1kKHb5LBfUQ6AEIKTAA\#v=onepage \&q=encuesta\&f=false}


Vol. 4, $\mathrm{N}^{\circ} 1$., p.06-22, enero - marzo, 2020

Badilla, E., Rojas, Wilfredo. y Vargas, I. (2008). Ubicación de sitios aptos para la disposición de desechos sólidos al oeste del valle central, Costa Rica. Revista Geológica de América Central, (38), 7-19. Recuperado de http://www.redalyc.org/articulo.oa?id=45437345001

Berenguer, M., Trista, J. y Deas, D. (2006). El reciclaje, la industria del futuro. Ciencia en su $P C$, (3). Recuperado de http://www.redalyc.org/articulo.oa?id=181322792005

Chaman, A. (1989), Manejo de la basura y su clasificación. Recuperado de http://biblioteca.usac.edu.gt/EPS/07/07_1989.pdf

Chassaigne, G. y Pinto, G. (2014). Determinación de variables que inciden en la estimación de residuos y desechos sólidos municipales recolectados en Venezuela. Interciencia, 39(12), 874-881. Recuperado de http://www.redalyc.org/articulo.oa?id=33932786008

Córdova, G. y Romo, Ma. de Lourdes. (2012). Gestión pública para solucionar un problema ambiental: manejo de llantas de desecho en Ciudad Juárez. Región y Sociedad, 24(53), 119151. Recuperado de http://www.redalyc.org/articulo.oa?id=10223040004

Empresa Municipal de Aseo de Cuenca. (2014). Emac: Cuenca Alcaldía. Recuperado de http://www.emac.gob.ec/?q=content/recolecci\%C3\%B3n-0

González, R. y Vega, R. (2002). Materiales de suelos de Yucatán factibles de utilizarse como cubierta en sitios de disposición de desechos sólidos. Revista Internacional de Contaminación Ambiental, 18(2), 57-66. Recuperado de http://www.redalyc.org/articulo.oa?id=37018201

Guevara, E. (2000). Diagnóstico de la situación ambiental y ecológica del Estado Carabobo. Revista INGENIERÍA UC, 7(1). Recuperado de http://www.redalyc.org/articulo.oa?id=70770104

Hernández, R., Fernández, C. y Baptista, M. (2014). Metodología de la investigación. México D.F: McGRAW-HILL / INTERAMERICANA EDITORES, S.A. DE C.V. Recuperado de http://observatorio.epacartagena.gov.co/wpontent/uploads/2017/08/metodologia-de-la-investigacion-sexta-edicion.compressed.pdf 
Vol. 4, $\mathrm{N}^{\circ} 1 .$, p.06-22, enero - marzo, 2020

Idrovo, E., Luna, K., Ordoñez, J. y Lituma, M. (2019). Marketing estratégico turístico para la Provincia del Azuay-Ecuador. doi: https://doi.org/10.33262/visionariodigital.v3i2.408

Limas, M. y Blanco, J. (2017). Prácticas de consumo-desecho de residuos sólidos domiciliarios en Ciudad Juárez en 2014. Iztapalapa, (83), 97-134. Recuperado de http://www.redalyc.org/articulo.oa?id=39357921005

Martínez, G., Tosca, C. y Juárez, A. (2019). Impuestos ambientales sobre los desechos sólidos en Tabasco: en pro del medio ambiente. Telos, 21(2). Recuperado de http://www.redalyc.org/articulo.oa?id=99359223018

Micucci, H., Jarne, A., Ferrarotti, N., Munitis, M. y Peruzzetto, C. (2005). Riesgo biológico en desechos sólidos y líquidos domiciliarios y de centros de atención primaria de salud. Acta Bioquímica Clínica Latinoamericana, 39(1), 43-57. Recuperado de http://www.redalyc.org/articulo.oa?id=53522191008

Orozco, C., Pérez, A., González, M., Rodríguez, F., y Alfayate, J. (2003). Contaminación ambiental: $\quad$ una visión desde la química. Madrid, España: Paraninfo. Recuperado de https://books.google.es/books?hl=es\&lr=\&id=nUoOx8knyUC\&oi=fnd\&pg=PA87\&dq=contaminaci\%C3\%B3n+ambiental\&ots=HIXbQHFeC L\&sig=D1iImjY7RFrjenVEKU96nfjxjKA\#v=onepage\&q=contaminaci\%C3\%B3n\%20a mbiental\&f $=$ false

Pacheco, M., Rodríguez, L. y Bermúdez, L. (2006). Plan estratégico de mercadeo para la cadena hortícola en el Distrito de riego del Alto Chicamocha. Agronomía Colombiana, 24(1), 182191. Recuperado de http://www.redalyc.org/articulo.oa?id=180316238020

Ramírez, M. (2005). Geopolítica de la crisis en la gestión de los desechos sólidos domésticos (gran área metropolitana de costa rica: 1950-2005). Revista de Ciencias Sociales (Cr), 3-4(109110), 101-125. Recuperado de http://www.redalyc.org/articulo.oa?id=15311008

Ramos, K. y Jiménez, Y. (2016). Degradación de desechos tóxicos provenientes de laboratorios universitarios con foto-Fenton solar usando diseño de experimentos. Revista Internacional de Contaminación Ambiental, 32(1), 119-131. Recuperado de http://www.redalyc.org/articulo.oa?id=37045275010 
ISSN: 2602-8506

Vol. 4, $\mathrm{N}^{\circ} 1$., p.06-22, enero - marzo, 2020

Rodríguez, A., Morales, M., Villarreal, E. y Quesada, A. (2016). Plan estratégico de

mercadotecnia para el desarrollo de una marca de la universidad tecnológica de la costa.

Revista Mexicana de Agronegocios, 39, 409-422. Recuperado de

http://www.redalyc.org/articulo.oa?id=14149188005

Salkind, Neil. (1999). Métodos de investigación. México: Pearson Educación. Recuperado de https://books.google.com.ec/books?id=3uIW0vVD63wC\&printsec=frontcover\&dq=meto dos + de + investigacion $\&$ hl $=$ es $\& s a=X \& v e d=0 a h U K E w j c 6 Z P 3 u-$

flAhXJ1FkKHRliBukQ6AEIMTAB\#v=onepage $\& q=$ metodos $\% 20 \mathrm{de} \% 20$ investigacion $\& \mathrm{f}$ $=$ false

Solano, O., Riascos, S. y Aguilera, A. (2013). Determinantes de los planes estratégicos de los sistemas de información en las pymes colombianas: caso Santiago de Cali - Colombia. $\begin{array}{llll}\text { Entramado, } & 9(1), & \text { 26-36. } & \text { Recuperado }\end{array}$ http://www.redalyc.org/articulo.oa?id=265428385003

Stutely, R. (2000). Plan de negocios: la estrategia inteligente. México: Prentice Hall. Recuperado de https://books.google.com.ec/books?id=QkxqKiF9TYoC\&pg=PA17\&dq=plan+estrategic o+de+negocios\&hl=es\&sa=X\&ved=0ahUKEwjEouWJ1o3mAhWsrFkKHdXwCm0Q6A EIMDAB\#v=onepage \&q=plan\%20estrategico\%20de\%20negocios\&f=false

Tamajón, C y Quevedo, F. (2007). Estudio del impacto ambiental de un colector solar de polipropileno. Ciencia en su PC, (4), 53-66. Recuperado de http://www.redalyc.org/articulo.oa?id=181320170006

Vélez, L. (2012). MODELO PARA ESTABLECER SANCIONES PECUNIARIAS PARA DELITOS AMBIENTALES. Revista Dyna, 79(175). Recuperado de http://www.redalyc.org/articulo.oa?id=49624958002

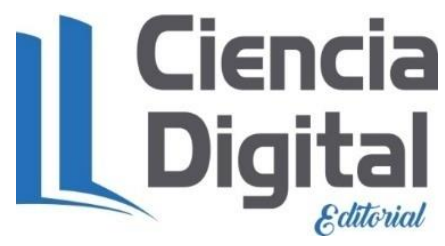


Vol. 4, $\mathrm{N}^{\circ} 1 .$, p.06-22, enero - marzo, 2020

\section{PARA CITAR EL ARTÍCULO INDEXADO}

Luna Altamirano, K. A., Yanza Nantip, J. G., Miguitama Gonzalez, D. E., \& Plasencia Sagbay, G. M. P. S. (2020). Plan estratégico para la contaminación ambiental en el sector del arenal de la ciudad de Cuenca-Ecuador. Visionario Digital, 4(1), 06-22. https://doi.org/10.33262/visionariodigital.v4i1.1064

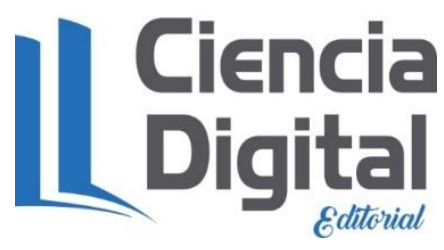

El artículo que se publica es de exclusiva responsabilidad de los autores y no necesariamente reflejan el pensamiento de la Revista Visionario Digital.

El artículo queda en propiedad de la revista y, por tanto, su publicación parcial y/o total en otro medio tiene que ser autorizado por el director de la Revista Visionario Digital.
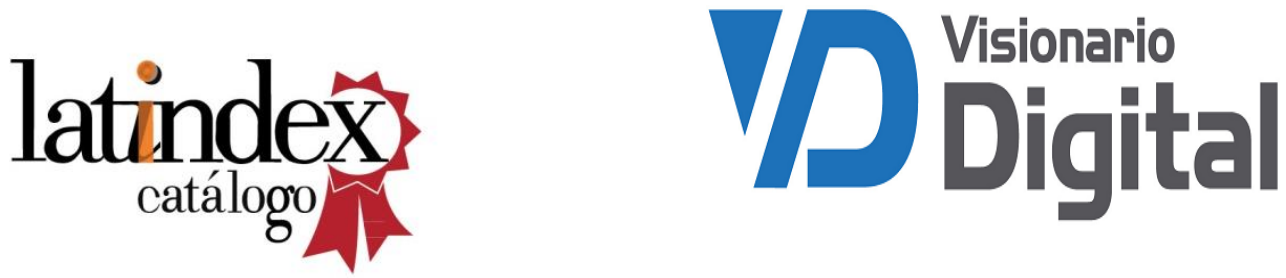